\title{
Correction to: Interpretability of Machine Intelligence in Medical Image Computing and Multimodal Learning for Clinical Decision Support
}

Kenji Suzuki, Mauricio Reyes, Tanveer Syeda-Mahmood, Ender Konukoglu, Ben Glocker, Roland Wiest (D), Yaniv Gur, Hayit Greenspan, and Anant Madabhushi

\section{Correction to: \\ K. Suzuki et al. (Eds.): Interpretability of Machine Intelligence in Medical Image Computing and Multimodal Learning for Clinical Decision Support, LNCS 11797, https://doi.org/10.1007/978-3-030-33850-3}

The chapter titled "Incorporating Task-Specific Structural Knowledge into CNNs for Brain Midline Shift Detection" was revised. The names of two authors were spelled incorrectly and the grant number was missing the final digit. This was corrected.

The original version of this book was revised. Due to an error, the volume editor's affiliation "ETH Zurich" appeared on SpringerLink instead of his name "Ender Konukoglu." This was fixed.

The updated version of the book can be found at https://doi.org/10.1007/978-3-030-33850-3_4 https://doi.org/10.1007/978-3-030-33850-3 